Pedagogía y Saberes No. 47

Universidad Pedagógica Nacional

Facultad de Educación. 2017, pp. 31-47

\title{
El análisis didáctico de Wolfgang Klafki como alternativa para la enseñanza de la filosofía
}

Artículo de investigación

Didactic Analysis of Wolfgang Klafki as an Alternative for Teaching Philosophy A análise didática de Wolfgang Klafki como alternativa para o ensino da filosofia

Diana Melisa Paredes Oviedo*

Para citar este artículo:

Paredes, D. (2017). El análisis didáctico de Wolfgang Klafki como alternativa para la enseñanza de la filosofía. Pedagogía y Saberes, (47), 31-47.

* Profesora del Instituto de Filosofía de la Universidad de Antioquia. Estudiante de Doctorado en Educación en la Universidad Pedagógica Nacional y del Doctorado en filosofía en la Universidad de Antioquia. Investigador del Grupo Formación y Antropología Pedagógica e histórica (Formaph) de la facultad de educación, Universidad de Antioquia. Correo: diana.paredes@udea.edu.co

Perfil ORCID: http:// orcid.org/0000-0001-5317-3480 


\title{
Resumen
}

El artículo es una reflexión que se desarrolla en dos partes: la primera de ellas expone los elementos esenciales de la propuesta de una Didáctica Críticoconstructiva de Wolfgang Klafki que tiene la formación como concepto clave para pensar la enseñanza, cuya meta esencial es lograr la emancipación de los estudiantes a través de la autodeterminación, la cogestión y la solidaridad. Lo anterior se materializa en pautas para un diseño didáctico concreto (análisis didáctico) y en su interés por impulsar escenarios educativos democráticos. La segunda parte es un ejercicio de análisis didáctico aplicado al campo de la enseñanza de la filosofía que ofrece a los docentes de filosofía un enfoque para el diseño de sus propuestas formativas teniendo como norte la emancipación y la transformación social.

\section{Palabras clave}

análisis didáctico; didáctica crítico-constructiva; enseñanza de la filosofía; planeación didáctica

\begin{abstract}
This article is a reflection made in two parts: the first one presents the essential elements of the proposal of a critical-constructive didactics of Wolfgang Klafki, where training is a key concept to approach teaching, with the essential goal of shaping independent students through self-determination, co-management and solidarity. This is materialized in guidelines for a concrete didactic design (didactic analysis) and in its interest to promote democratic educational scenarios. The second part is an exercise of didactic analysis applied to teaching philosophy that offers to philosophy teachers an approach for the design of their education proposals with the final goal of achieving independence and social transformation.
\end{abstract}

\section{Keywords}

didactic analysis; critical-constructive teaching; teaching of philosophy; didactic planning

\section{Resumo}

0 artigo é uma reflexão que se desenvolve em duas seções: a primeira delas expõe os elementos essenciais da proposta de uma Didática Crítico-construtiva de Wolfgang Klafki, na que a formação é conceito básico para pensar o ensino, cuja meta essencial é conseguir a emancipação dos estudantes através da autodeterminação, a congestão e a solidariedade. Isso é evidente nas pautas para um planejamento didático concreto (análise didática) e no seu interesse por impulsar cenários educativos democráticos. A segunda seção é um exercício de análise didática aplicado ao campo do ensino da filosofia, que oferece aos professores de filosofia uma abordagem para o planejamento das suas propostas formativas baseadas na emancipação e a transformação social.

\section{Palavras chave}

análise didática; didática crítico-construtiva; ensino da filosofia; planejamento didático 


\section{Parte uno. La base humanista de la didáctica crítico-constructiva de Wolfgang Klafki y su propuesta de análisis didáctico}

L a propuesta pedagógica de Wolfgang Klafki tuvo dos momentos, el primero acontece antes de 1960 y luego de este periodo da un giro hacia una perspectiva crítica; cabe anotar que su interés por la formación y el acontecer escolar se ha mantenido a lo largo de ambos. En 1963 escribió la obra Estudios sobre la teoría de la formación y la didáctica (1975) en la que hizo evidente el cambio que operaba en su pensamiento. Allí planteó que los rasgos de la pedagogía y la didáctica humanista, especialmente su enfoque histórico-hermenéutico, resultarían cruciales para una propuesta didáctica que buscara concretar las orientaciones de las ciencias generales crítico-constructivas de la educación.

La perspectiva didáctica humanista, como disciplina de la pedagogía humanista, se enfocaba en la enseñanza y respondía a la filosofía de la vida propia de las ciencias de la educación y de la ciencia humanista del siglo XIX apoyada, a su vez, en los trabajos de Wilhelm Dilthey. En este sentido, esta propuesta se inscribe en la tradición europea del pensamiento pedagógico que va desde Rousseau, Pestalozzi, Humboldt, Herbart, Schleiermacher hasta Fröbel. El desarrollo y acogida de esta didáctica oscila entre 1918 y se extiende hasta mediados de 1960, pero será la tercera generación de este movimiento la que se encamine hacia la perspectiva crítico-constructiva. Los elementos de la didáctica humanista que recoge Klafki los expuso con suma claridad Erich Weniger (1962) y son los siguientes:

1. La didáctica incluye o presupone decisiones orientadas hacia un fin o una propuesta en el marco de una idea de formación.

2. Las decisiones didácticas, que se materializan a través de la selección y estructuración de los objetivos y contenidos para la enseñanza, son fruto de decisiones históricas bajo ciertas condiciones, a su vez, históricas.

3. Las decisiones didácticas son tomadas en el marco de luchas entre poderes políticos, espirituales y sociales; se materializan en las tensiones entre pedagogos y políticos, quienes usualmente toman decisiones sobre objetivos didácticos.

4. El Estado es responsable del currículum, pero su responsabilidad se demarca desde el impulso a la autonomía en la educación que busca capacitar a los jóvenes para que tomen decisiones propias y futuras, y así se conviertan en mayores de edad.

5. Siguiendo a Rousseau, comprende cada etapa de la vida humana como valiosa en sí misma y necesaria para la realización de la siguiente. En este sentido, la educación responde a las necesidades del presente y a los deseos del futuro de los jóvenes.

6. La investigación y la teoría didáctica son vistas como ayudas ilustradoras para la toma de decisiones que darán forma al currículum y a los reglamentos; también cumplen la misma función para los profesores y su necesidad de solucionar las tareas didácticas en la clase.

7. Se pone en escena la discutida idea de que la didáctica, en cuanto se refiere a las decisiones sobre objetivos y contenidos, prima frente a la metodología.

8. Los anteriores son principios didácticos que operan para la didáctica especial de cierto tipo de escuela o materia o grado. Las decisiones didácticas no se deducen de la materia de conocimiento: hay diferencias entre las ciencias de la materia con la materia escolar. La finalidad de esta última es transmitir una percepción elemental de la realidad y del propio yo, por ello las decisiones didáctica no las toma la materia respectiva, pero sí se toman en relación con ella. Se propone entonces un campo en el que las didácticas de las materias se desarrollen como disciplinas científicas e independientes, aunque con un vínculo estrecho con las ciencias de la educación, la didáctica general y las ciencias de las materias.

9. La didáctica o la pedagogía científica son vistas como ciencias de la praxis para la praxis, a lo que se agrega que están imbricadas en la responsabilidad por la próxima generación.

10. En general, la didáctica humanista operó a partir de un procedimiento histórico hermenéutico. Weniger y Flitner, esencialmente, se acercaron a los documentos didácticos con el fin de aclarar situaciones sobre las decisiones didácticas, encaminándose a posibilitar y pedir decisiones pedagógicas conscientes y fundadas. Es necesario precisar que por "documentos didácticos" se entiende textos de diferentes tipos: currículums, reglamentos, planeaciones de clase, propuestas didácticas, listas de objetivos, discusiones didácticas e ideas didácticas.

Klafki inicialmente es visto como un didacta humanista que asume la Pedagogía como una ciencia del espíritu, pero bajo el amparo de las ideas de autores como Theodor W. Adorno, Herbert Marcuse, Max Horkheimer y Jürgen Habermas, se adscribe a una 
perspectiva crítica que, en general, niega las condiciones injustas e indeseables de la sociedad imperante y lucha contra toda forma de opresión social que impida la felicidad, la libertad y el orden racional de la sociedad. Si bien este cambio se va gestando y, paulatinamente, el pedagogo se mueve hacia una didáctica crítica, su interés por este campo se avizoraba desde su tesis doctoral de 1957 sobre El problema pedagógico de lo elemental y la teoría de la formación categorial (Klafki, 1972) en la que propuso una reflexión sobre la didáctica en sentido amplio, que le acompañará en sus siguientes trabajos, frente a una comprensión en sentido estricto de esta. Desde ese momento, el autor defendió su idea de que la didáctica, en sentido amplio, comprendería, además de la teoría de los contenidos de la formación, la metodología y la descripción y el análisis de todos los procesos de aprendizaje y enseñanza no planificados y no reflexionados. Es necesario reiterar que Klafki logró también, a lo largo de su trabajo, posicionar los contenidos de la formación en el centro del pensamiento pedagógico apelando a tres razones: a) la didáctica prima sobre la metodología. Antes de definir los métodos se requiere aclarar los contenidos y fines del proceso formativo con base en los que se evalúa la pertinencia de los primeros. b) La formación se resuelve a través de los contenidos de la cultura, es decir: los contenidos son medio y fin. c) Las decisiones didácticas son históricas y responden a las demandas de la época, es por ello que el didacta siempre tendrá que redefinir sus decisiones fruto de los retos a los que se enfrenta. Serán estas ideas las que impulsarán a este pedagogo en sus siguientes búsquedas asociadas con el contenido de la formación. Su esfuerzo estará atravesado por dos preguntas: ¿Cómo reducir los contenidos disciplinares a contenidos formativos? ¿De qué manera se vincula el contenido formativo a la condición actual y a la condición futura de los estudiantes? Klafki apoya el giro realista para la investigación pedagógica en Alemania y se orienta, paulatinamente, a investigaciones en las que se combinan lo filosófico, lo pedagógico y asuntos concretos de la escuela, exponiendo su idea de la unidad entre teoría y praxis.

Continuando con la reconstrucción, en un curso radiofónico y en un artículo de 1971 titulado La ciencia de la educación como teoría crítico-constructiva: hermenéutica-empírica-crítica de la ideología el autor desarrolla su propuesta de crítica de la ideología vinculada a la educación. Se pregunta por cómo los fines de la educación son construidos y por la forma en que estos se encuentran comprometidos, consciente o inconscientemente, con los intereses de ciertos grupos o sectores sociales; siguiendo su línea discursiva, se hace necesario revisar la ideología en dos niveles: el primero se enfoca en el análisis de los objetivos educativos y la existencia de posibles intereses sociales no reflexionados y el segundo en la existencia o no de estrategias intencionales de ciertos grupos, cuyo fin sea el ocultamiento de los intereses particulares y la defensa de una conciencia falsa detrás de ellos. Para lograr este cometido la hermenéutica, la perspectiva empírica y las críticas de la ideología son el apoyo procedimental, pues no se trata de una mera descripción de las metas educativas sino de un develamiento de lo que las sustenta.

En el corazón de este proyecto se encuentra la formación categorial, heredera de la tradición de la Bildung, en la que se asume la existencia de la transmisión entre el sujeto y el objeto abierta hacia adelante; lo anterior se traduce en una apropiación activa por parte del sujeto, a quien se le abre la realidad histórica y quien se abre a la realidad. Para Klafki la formación es un derecho que tienen todos los seres humanos, incluye todas las posibilidades humanas que ayuden a la autogestión y el desarrollo de otros seres humanos, y se realiza por medio de lo universal (aquello que nos concierne a todos, por ejemplo las tareas, problemas, pensamientos y soluciones comunes). Lo que importa es ofrecerles a los jóvenes las condiciones para que se apropien de la historia, teniendo como meta que configuren su presente y su futuro de manera autónoma.

Situarse en lo crítico implica, para este autor, reconocer que la didáctica está orientada a la capacitación de niños, niñas, jóvenes y adultos, a través de ayudas didácticas, para una creciente capacidad de solidaridad, auto y cogestión. También exige asumir que muchos contextos educativos no se ajustan a esta demanda, por ello el llamado es a procurar que sean cada vez más democráticos y que se logre intervenir lo que obstaculiza la meta expuesta, en virtud de lo cual la didáctica adquirirá un compromiso vital: indagar acerca de las causas que dificultan el desarrollo de la capacidad de solidaridad, auto y cogestión en el aprendizaje y la enseñanza, así como realizar, buscar, diseñar e intentar el desarrollo de tales procesos de enseñanza y aprendizaje.

Frente al término constructivo, Klafki se refiere a la relación de la didáctica con la praxis más continua y al interés por la realización, la configuración y los cambios de esta. Va más allá de la demanda de aclaración de la conciencia práctica sobre las condiciones, las posibilidades y límites del actuar pedagógico, e incluye las anticipaciones de la teoría, los modelos para una praxis posible, los conceptos esenciales para una praxis escolar y una enseñanza de corte democrático, así como para nuevas maneras de correlación entre 
praxis y teoría. En consecuencia, la praxis didáctica y su teoría se ocupan, de acuerdo con mi análisis de la propuesta klafkiana, de lo siguiente: a) los métodos de la enseñanza y el aprendizaje. b) Los medios de la enseñanza y aprendizaje que se humaniza al considerar las condiciones de aprendizaje de los estudiantes. c) Las medidas de control, evaluación y sanciones que influyen en estudiantes y profesores. d) La elección de contenidos o temas de la enseñanza y aprendizaje orientados a ciertos fines. e) Las decisiones y argumentaciones sobre objetos generales y especiales de la enseñanza y el aprendizaje. f) Los procesos pedagógicos realizados en los campos específicos (acordados y ocultos).

El didacta crítico-constructivo determinará los objetivos didácticos considerando el significado del contenido y la configuración interna de los fines formativos. Para lograr esta delimitación se apoyará, por un lado, en la investigación sobre la socialización en general, especialmente en aquella que se ocupa de los mundos cotidianos, sus influencias funcionales, sus procesos de aprendizaje, y su impacto en las opiniones, juicios, capacidades, conocimientos y esperanzas de los individuos. Por otro, se sirve también de la investigación institucional en la que se indaga sobre las estructuras, los reglamentos, la aceptación de los cambios en los procesos de enseñanza y aprendizaje en los colegios, universidades, casas de juventud, escuelas de enseñanza popular, sindicatos, etc.

Esta propuesta comprende entonces una amplitud en sus objetivos porque, si bien el autor recogió el concepto de Bildung propio de la tradición alemana, tal como se había indicado en el desarrollo de su trabajo, a partir de 1970 se enfocó en la relación teoría-praxis en el seno de una ciencia crítica de la educación y las consecuencias de ello en el proceso formativo. La imagen de humanidad por la que luchan los pedagogos y didactas inscritos en esta línea es una humanidad madura, libre, y con capacidad de autodeterminación, democratización y emancipación. Se trata de seres humanos que determinan sus acciones racionalmente, que se ocupan de reconocer libremente a los otros seres humanos y con las posibilidades para su individuación, lo que late aquí es la defensa de la emancipación tanto social como individual en cuanto condición humana, y la configuración de esta ciencia crítica de la educación como una teoría crítica de la sociedad y sus formas de humanización.

En conclusión, la propuesta didáctica de Klafki (1985a) se encuentra enmarcada en su concepción de la pedagogía desde el enfoque crítico, cuya nota fundamental es que no se debe partir de supuestos teóricos sino de la realidad educativa para pensar tanto la enseñanza de saberes como la formación de los sujetos en términos generales. En otras palabras, podríamos afirmar que la pedagogía debe considerar de manera explícita las problemáticas sociales, que serán abordadas metodológicamente desde la empiria, la crítica ideológica y la hermenéutica, con miras a adelantar una propuesta de investigación que permita descubrir las formas de falsa conciencia que influyen de manera subrepticia sobre los individuos y que determinan las maneras de enseñar y aprender.

\section{Lo elemental, fundamental y ejemplar en la propuesta didáctica}

En la enseñanza hay que establecer contenidos formativos que, habida cuenta de las respectivas edades, forman la conciencia y que vienen a ser formativos de su pensar y de su actitud, se plantea entonces la siguiente pregunta: ¿De qué manera los educandos pueden en la enseñanza apropiarse de los contenidos culturales elementales? Cuando le mostramos al estudiante los contenidos concretos, se le posibilita reconocer el general implícito en ello, lográndose así la llamada interacción con el mundo: la realidad se abre al estudiante y el estudiante se abre a la realidad; a partir de tal proceso el estudiante adquiere categorías o conceptos fundamentales que sirven como presupuestos y principios de conocimiento y de comprensión del mundo que puede utilizar y aplicar de manera autónoma. Al respecto Klafki (1975) argumenta: "El devenir visible de los 'contenidos generales', de los principios categoriales en el 'material' paradigmático, es decir, de lado de la realidad, no es otra cosa que la consecución de 'categorías' del lado del sujeto" (p. 43). Los contenidos que forman en ese sentido y transmiten categorías como las planteadas, el autor los denomina elementaria ${ }^{1}$.

1 Los elementaria son contenidos básicos y fundamentales, a partir de los cuales el sujeto categoriza el mundo; el autor propone siete formas de lo elemental:

I. Lo fundamental, que se puede experimentar solo en términos de vivencia. Las experiencias y vivencias fundamentales solo se pueden desplegar no definir, ocurren en situaciones específicas no necesariamente planeadas, teniendo como sustrato el hecho de que aparecen estados de ánimo difíciles de ser conceptualizados.

II. Lo ejemplar, que se entiende como aquello según lo cual algo general se experimenta con algo particular, en otros términos: lo general se abstrae de lo particular.

III. Lo típico a partir del cual lo general se experimenta en lo particular y se nos revela como tipo.

IV. Lo clásico, donde lo general se experimenta a partir de un valor o valoración.

V. Lo representativo, según lo cual lo general se experimenta como actualización, como presentificación.

VI. Las formas finales simples en donde lo general se aprende en un hacer o realización de lo particular, sobresaliendo aquí el carácter práctico. 
En la situación dialéctico-formativa del sujeto y el mundo, Klafki reconoce tres dimensiones que sirven para solucionar la pregunta por la selección de los contenidos: fundamental, elemental y ejemplar. La primera se refiere a los contenidos, categorías y principios más generales que, se espera, sean apropiados por el educando de modo experimental. La dimensión elemental concierne a los contextos, conocimientos y procedimientos en los que, gracias al ejemplo, se hace visible y reconocible un principio válido que es aplicable a muchas otras cosas. La dimensión ejemplar se centra en que lo elemental y lo fundamental se consigan mediante un buen ejemplo que deje una impresión profunda. Lo ejemplar son contenidos que no solo existen para sí, sino que se abren o permiten abrirse a otros contenidos, le dan una estructura al campo de aprendizaje y se busca un orden en la cabeza de los educando, en otras palabras: podríamos señalar psicológicamente la presencia de conceptos básicos que nos permitan la adquisición de conceptos más elevados que nos llevan a niveles de complejidad diferentes. Klafki (1972) le prestó mucha atención a la dimensión elemental, puesto que en ella se pone en juego la mediación y transmisión de experiencias fundamentales y de apreciaciones al educando que hacen posible, mediante la experiencia, reconocer las cosas, pues abre el mundo al hombre y el hombre al mundo, y es sucinto y sintético ya que, por una parte se relaciona con el presente y el futuro del educando, y por otra, se expresa en lo general y en lo particular.

Si tomamos un contenido de enseñanza y lo observamos a la luz de esas tres dimensiones pedagógicodidácticas, se justifica el análisis de la estructura del contenido. En términos de la formación, los aspectos pedagógicos no se encuentran supeditados a las dinámicas mismas de los contenidos (objetivo), sino que los parámetros didáctico-formativos son los que guían tales cuestionamientos. La planeación didáctica va primero y está atravesada por las dinámicas propias del saber a enseñar y de los fines formativos asociados al proceso de enseñanza, orienta el contenido y el método de transmisión. Los principios didácticos delimitan los contenidos de la enseñanza al punto que nos permiten señalar qué contenidos deben ser enseñados y cuáles no, lo anterior en virtud de su potencial formativo y emancipatorio.

La propuesta de Klafki (1985a) en este punto se orienta hacia las investigaciones sobre el análisis didáctico que hace posible una reducción didáctica. Este

VII. Las formas estéticas simples en donde lo general se vuelve visible mediante lo único. Podemos experimentar lo general en algo concreto que se nos revela como experiencia única. análisis consiste en situar las preguntas necesarias para la selección, la cualidad y la estructura de los contenidos de la formación en el corazón de la preparación de las clases, eso significa que no queda en manos de las dinámicas de los contenidos en sí mismos. Por un lado, entonces, tenemos a las ciencias o disciplinas que ofrecen información y competencias con relación a los contenidos y, por otro, tenemos a la didáctica que crea las condiciones para que ese objeto o contenido se transforme en contenido formativo, en sentido de una formación categorial que incluya lo material y lo formal, el sujeto y el mundo. Todo lo anterior muestra cómo la propuesta didáctica de Klafki reinterpretó de un modo didáctico el concepto neohumanista de Bildung, logrando que aparezca como útil para la enseñanza. El análisis didáctico se entiende como la interpretación y estructuración didácticas con miras a una planeación de la enseñanza.

Todo este andamiaje responde a la combinación de enfoques de investigación en su perspectiva de la Ciencia de la Educación. La mezcla de la hermenéutica, la investigación empírica y la crítica ideológica se constituye en una síntesis necesaria para poder afrontar el reto de la emancipación de los educandos. La hermenéutica ofrece el suelo interpretativo para los resultados que aporta la investigación empírica, mientras que la crítica ideológica brinda a la hermenéutica un análisis de las condiciones sociopolíticas de una época que harán posible enfrentar el statu quo. Este marco de investigación ampliado permite revisar el comportamiento de la praxis pedagógica y ofrecer orientaciones para su diseño, fruto de experimentos.

\section{Componentes del análisis didáctico}

La didáctica se enfoca entonces a ofrecer un escenario para modelar una praxis educativa en la que se fortalece el aprendizaje formativo del alumno, quien desarrolla aptitudes, capacidades y conocimientos efectivos a través de un número limitado de ejemplos elegidos para elaborar, a su vez, conocimientos, capacidades y actitudes que se pueden generalizar. Este tipo de aprendizaje es categorial, pues le facilita al estudiante adquirir un andamiaje que le ayudará, por analogía, a enfrentar las situaciones nuevas en las que se encuentre en diferentes contextos; podría afirmar que de lo que se trata en este proceso es de adquirir una comprensión de las cosas que simultáneamente es una estrategia para establecer relaciones, responder cuestionamientos y planear acciones. Tenemos entonces, de un lado, la enseñanza vista como una ayuda para el aprendizaje autónomo y, de otro, el aprendizaje ejemplar como autónomo y genético puesto que reconstruye y descubre los contenidos. 
El pedagogo alemán sugiere al didacta revisar las dimensiones que intervienen en el proceso de aprendizaje y el modo en que se vinculan con los temas generales, significa entonces que Klafki (1991) pone en el centro de la planeación los contenidos de enseñanza y no los métodos. De lo que se trata en la situación formativa es de presentar los contenidos desde una perspectiva científica en la que se realiza un esfuerzo por transmitir los modos fundamentales del entendimiento, la manera en que los métodos de la ciencia se ponen en funcionamiento, dejando de lado cualquier pretensión de situarlos solo en un plano teórico y revelar el decurso histórico de un saber mostrando la correlación entre la teoría y la praxis. En la clase no se exponen contenidos a secas, los contenidos educativos se deben analizar a partir de sus contenidos formativos, ese es el análisis didáctico, y son revisados desde cinco preguntas básicas que le ayudan al maestro a cuestionarlos de manera crítica.

Todo educador que pretenda trabajar con contenidos educativos o de enseñanza, desde una perspectiva formativa, debe analizarlos de acuerdo con estas cinco preguntas, y si soportan este ejercicio se legitima la enseñanza de estos bajo los parámetros teórico-formativos; en ese sentido, si la respuesta a alguna de esas preguntas es negativa significa que no cuenta con el potencial formativo requerido. Las preguntas didácticas propuestas por Klafki, según parte del análisis que hace de ellas Andrés Runge (2008), son las siguientes:

a. El significado ejemplar del contenido: ¿Qué contexto general de sentido o del mundo soporta o abarca ese contenido? ¿Qué fenómeno fundamental, principio básico, criterio, problema, método, técnica, actitud se puede comprender de una manera ejemplar a partir de la relación con ese contenido? ¿De qué debería ser ejemplar, representativo, típico de ese tema? ¿En dónde se puede hacer fructífero posteriormente el aporte de ese tema en cuanto totalidad o en elementos particulares (apreciaciones, representaciones, conceptos valorativos, métodos de trabajo, técnicas)? Se trata de una pregunta en la que revisa la manera en que ese principio general se manifiesta en situaciones específicas; así, se identifica lo fundamental del contenido y lo que representa como un horizonte de comprensión amplio para el educando. Considero necesario apelar a un ejemplo para aclarar esta pregunta: tomemos como tema el ejercicio del voto en las escuelas colombianas en las que existe algo denominado gobierno escolar. ¿De qué es representativo el tratar el tema del voto en la escuela? Esta acción manifiesta una idea de participación en la que todos tienen un lugar, da cuenta de unos mecanismos para la toma de decisiones en comunidad y, quizá, de cierta forma de gobierno. Siguiendo a Klafki, lo general de esta acción, más allá de la mecánica electoral, lo que expone es una visión de la vida en comunidad.

b. Importancia para el presente: $i Q u e ́$ importancia tiene el respectivo contenido en la vida espiritual del niño o del educando? ¿Qué importancia debería tener tal contenido desde el punto de vista pedagógico? Klafki se preocupa por la artificialidad de muchos contenidos escolares y que, sin pretenderlo, someten a la inutilidad a muchos contenidos disciplinares. Su apuesta es por saber si el contenido que se presenta a los educandos tiene algún significado en su vida presente. Retomando el ejemplo de la pregunta anterior, el voto en la escuela y el gobierno escolar, le corresponde al didacta preguntarse si este tema cumple algún papel en el presente del educando. En este caso podríamos afirmar que sí, porque le permite apropiarse de unas formas de participación y cogestión que se articulan a ciertas perspectivas democráticas.

c. Importancia para el futuro: $i$ En qué consiste la importancia del tema para el futuro del niño o del educando? Se debe exigir que la enseñanza explique las cosas y fortalezca a niños y a jóvenes para que logren actuar de forma adecuada bajo las características de un futuro incierto. Wolfgang Klafki sugiere que el profesor actúe como un profano formado, como alguien que se hace una idea de los conocimientos y capacidades que necesitaría un ciudadano maduro para enfrentar diferentes roles y situaciones críticas. Hay un marcado interés espiritual y social por las condiciones de ese adulto que probablemente tendrá una familia, un empleo, circulará por una ciudad, etc. En el caso de nuestro ejemplo, el didacta se preguntará por las posibles dificultades del educando en los diferentes momentos en que deba votar: compra de votos, mal uso de la participación, tipos de mayoría requerida para atender una votación, etc.

d. Estructura del contenido: $¿$ Cuál es la estructura del contenido, según las preguntas pedagógicas de la primera, segunda y tercera dimensiones? ¿Cuáles son los momentos particulares del contenido y en qué conexión se encuentran esos momentos particulares? ¿En una conexión lógica, fáctica? ¿Tiene niveles el contenido? ¿Tiene diferentes niveles de sentido y de importancia? ¿Pueden ser comprendidos esos niveles de una manera independiente entre sí? ¿En qué contexto mayor se encuentra ese contenido? ¿Qué propiedades del contenido podrían hacerle difícil al educando su apropiación? ¿Qué se tiene que mantener como saber mínimo para que el contenido pueda ser tenido como un contenido espiritual vivo y efectivo? Esta pregunta depende de las tres 
anteriores y supone una elección del didacta frente al orden en que presentará el contenido a los estudiantes, de manera que, por un lado, se apropien del principio general a través de ejemplos horizonte de comprensión del mundo-y, por otro, fortalezcan su actitud crítica desde esta perspectiva formativa. Continuando con el ejemplo de la democracia, el didacta podría empezar su trabajo con la siguiente pregunta: $¿$ Ha sido siempre aceptada la democracia como forma de gobierno deseable? En este caso el didacta opta por un enfoque en el que se desnaturaliza la democracia y se compara con otras formas de gobierno, se revisan sus transformaciones históricas y también las posturas a favor y en contra; con ello se impulsa una apropiación crítica de este contenido por parte del educando, quien probablemente ganará elementos para revisar la constitución histórica de otros fenómenos sociales, lo anterior se irá especificando hasta llegar a la democracia escolar.

e. Asequibilidad del contenido: ¿Cuáles son los sucesos, situaciones, intentos a partir de los cuales los niños de esa clase, de ese nivel pueden, de una manera interesada, cuestionar, comprender, contrariar, intuir, acceder a la estructura del respectivo contenido? ¿Cuáles son las situaciones, observaciones, intuiciones, intentos, modelos apropiados para que los educandos den respuesta de una manera autónoma sobre la estructura de ese contenido? ¿Cuáles son las situaciones, observaciones, intuiciones, intentos, modelos apropiados para que con un ejemplo, con un caso elemental se haga productivo el principio de una cosa, la estructura de un contenido y se puede aplicar y a partir de ahí ejercitar? Lo central en esta pregunta es acercarse al modo como los jóvenes se apropian del contenido, en otras palabras: que las estrategias de enseñanza y aprendizaje cuenten con un anclaje en la generación en formación. Nuestro ejemplo de la votación y la democracia no podrá ser presentado solo con la típica estrategia del tarjetón, las fallas en su diligenciamiento, sino que deberá recurrir a otras experiencias: redes sociales (Twitter, fanpage, Facetime, posicionamiento de marca, espacios colaborativos, etc.).

El análisis didáctico trata entonces de cerrar las brechas entre las teorías de la formación formal y la formación material a la base de las didácticas de su época. Aquí se parte de la complementariedad de ambas perspectivas, pero se propone un orden: precede el trabajo con los contenidos de las disciplinas, prosigue el análisis didáctico y se cierra con las propuestas metódicas para la enseñanza. El profesor o didacta para la preparación de sus clases, que prefiero nombrar como proyecto o propuesta formativa, y para la definición de los métodos de enseñanza, tendrá en cuenta, según Klafki (1970), en primer lugar, los objetivos de formación y los contenidos; en segundo lugar, el tipo de clase al que se enfrenta (frontal, individual, en pareja, en grupo, etc.); en tercer lugar, las formas de enseñanza y aprendizaje del educando; por último, las condiciones técnicas y los medios de la enseñanza.

\section{Parte dos. Apropiación de los elementos del análisis didáctico de Wolfgang Klafki en la enseñanza de la filosofía}

Desde el enfoque de este autor, la clase es vista como un proceso social. En ella se encuentran las biografías de los profesores y estudiantes, así como las condiciones del contexto como modeladoras del encuentro. La línea operativa de esta propuesta didáctica es aprender y enseñar, a través de modelos ejemplares, parte de la existencia de problemas esenciales en las disciplinas, que serán presentados históricamente y luego se exploran las alternativas de solución a estos problemas; con base en este modo de proceder se incita al desarrollo de las capacidades generalizables del alumno a partir de una serie de ejemplos seleccionados por el didacta. Todo objetivo o contenido que haya sido elegido para un objetivo pedagógico se convierte en un tema que sintetiza, a su vez, el contenido y el objetivo. De un lado, las preguntas del didacta y el interés de los estudiantes hacen que ese contenido devenga tema con carácter formativo; de otro, también el uso de los ejemplos que muestren el valor del contenido para la vida a través de la experiencia logra tal fin, por ello los temas o son métodos para crear posibilidades o son el resultado de procesos de búsqueda que siguen ofreciendo alternativas a los educandos. Lo anterior hace que todo tema que sea presentado en la clase tenga un carácter metódico implícito y sienta las bases del carácter inmanentemente teórico de la enseñanza.

Se aprende, desde el punto de vista del pedagogo alemán, por medio de temas ejemplares. Lo adquirido se consolida a través de ejemplos análogos a los ejemplos originales en la conciencia de los alumnos, quienes los transforman en capacidades referentes a los contenidos. No obstante, es necesario precisar que Klafki (1985a) sigue a Bruner en su desarrollo del aprendizaje ejemplar, y acepta que: a) el proceso de aprendizaje se entiende como una confrontación con la realidad y una adquisición de esta. b) Este proceso es tripartito e implica el trato directo y activo de la realidad, la mediación icónica y la mediación abstracta. c) Si bien hay una perspectiva evolutiva 
en cuanto al aprendizaje, los niveles de asimilación y adquisición tendrán énfasis en diferentes edades sin que se excluyan de las siguientes, por ejemplo siendo adultos lo icónico sigue resultando relevante y necesario. d) Uno de los problemas del aprendizaje en la escuela es obviar el trato directo y lo icónico e introducir prematuramente lo abstracto.

En el caso de la filosofía, siguiendo los elementos de Klafki, para pensar en la modelación de un encuentro formativo y en el diseño de una unidad didáctica o propuesta formativa, se requiere tomar una serie de decisiones que preceden el análisis didáctico y que enumero a continuación:

\section{¿Cuál es mi perspectiva filosófica?}

Es esencial situarse en una perspectiva para entender la filosofía. Como se ha expuesto, el didacta debe conocer la dinámica del contenido (paradigmas, tensiones, transformaciones, etc.) que pretende transformar en contenido formativo. Este es un primer momento para la definición de los temas, ejemplos y fines formativos, pues sitúa al analista didáctico en un lugar desde el que puede enfrentarse al contenido general de la disciplina.

Es necesario acoger enfoques de la filosofía que se ajusten a la perspectiva formativa de Klafki. Recomiendo dos perspectivas que cumplen con esta condición: la primera de ellas la ofrece Richard Rorty (2010), en La filosofía y el espejo de la naturaleza, obra en la que expone su distinción entre una filosofía sistemática y una filosofía edificante. Para llegar a ello parte de una reflexión sobre el discurso y encuentra que en toda sociedad se impone un discurso normal, entendiendo por este el que encarne los criterios aceptados y es donde encuentra su lugar la filosofía normal o sistemática que, según el autor, tiene una matriz cartesiano-kantiana.

Del filósofo pragmatista se puede retomar su concepción de filosofías edificantes, entendidas como aquellas que son anormales, que no se atienen a la tradición y sirven de ayuda para la revolución y transformación de paradigmas, algunos de los autores que ubica allí son: Ludwig Wittgenstein, Martin Heidegger y John Dewey. Si se toma a Wittgenstein y su trabajo filosófico como marco de la planeación didáctica, se parte del hecho que es visto como un filósofo edificante porque, precisamente, con su propuesta sobre el lenguaje lleva al traste los proyectos esencialistas y muestra que incluso las intuiciones se construyen en el seno de una cultura asentada lingüísticamente. Si optamos por Heidegger aceptamos que la pregunta por el ser y el tiempo es, en sí, una suerte de exigencia a la filosofía para que dé cuenta de lo que ha significado históricamente esta pretensión. El trabajo del filósofo es preguntarse por el ser y por este camino se descubre en el lenguaje y la historia. Por último, la perspectiva deweyana es anticartesiana y antifundacionalista. Aquí la filosofía es acción, no cuenta con un fondo de conocimiento propio y no es capaz de inventar mundos de la nada. Se agrega a esto que defiende un modo de vida democrático que transforma la educación en una experiencia de formación de hábitos inteligentes de acción.

La segunda perspectiva a acoger es aquella en que la filosofía es entendida como filosofía práctica. Gabriel Arnaiz (2007) plantea la existencia de un campo de investigación en filosofía que apunta a una concepción de esta como algo práctico y estrechamente vinculado con el cuidado de sí, entre los autores de ese campo se encuentran: Pierre Hadot, Michel Foucault y Michel Onfray. En esta clasificación los autores están interconectados, pues Hadot es tanto el referente de Foucault como de Onfray, lo que tendría en común es la defensa de una vida filosófica que rompe con las dinámicas de una filosofía como saber académico. Para Pierre Hadot (2009) la tarea del filósofo era cambiar su propia vida, pues los cursos o escritos que hacía resultaban secundarios y tenían como fin mantener la vida filosófica o ayudar a sus discípulos. La filosofía aparece como una terapéutica constante que busca la autonomía y la libertad interior. En el caso de Foucault (2008), este pone en el corazón de la filosofía una vuelta de tuerca sobre el cuidado de sí y no el conocimiento de sí como aquello que conduciría la vida de los hombres. Finalmente, la meta de Onfray (2008) ha sido resucitar de manera efímera el "Jardín de Epicuro" como forma de microrresistencia. Se trata de una filosofía materialista, epicúrea, hedonista, libertaria y libertina. Aunque Onfray está empeñado en la defensa de un modo de vivir ateo.

\section{¿Desde qué paradigma para la enseñanza de la filosofía puedo situarme?}

El didacta debe contar con una perspectiva desde donde oriente la selección de fines educativos, tal como menciona Klafki (1995). No se trata de una camisa de fuerza sino de un marco que acople una perspectiva filosófica con una pregunta por la finalidad formativa asociada a su enseñanza. Según Michel Tozzi (2003) existen cuatro paradigmas para pensar la enseñanza de la filosofía: a) histórico, b) doctrinario, c) problematizante y d) praxeológico. He nutrido las propuestas de este autor y presento un panorama ampliado. Por la elección filosófica realizada en el 
apartado anterior es necesario articularse con una propuesta de orden didáctico que sea coherente. Siguiendo a Tozzi (2003) los dos paradigmas que se ajustan son el problematizante y el praxeológico, a continuación presentaré las propuestas que constituyen cada paradigma.

Tabla 1. Perspectivas del paradigma problematizante para la enseñanza de la filosofía

Paradigma problematizante

La filosofía es tratada como un escenario en el que se cultivan la pregunta y el deseo de saber de maestros y estudiantes. Se encuentran las propuestas de Larrauri (2001) y de Kledzik (1992).

\begin{tabular}{|c|c|}
\hline $\begin{array}{l}\text { Una didáctica de } \\
\text { la filosofía como } \\
\text { educación filosófica }\end{array}$ & $\begin{array}{l}\text { Una didáctica de } \\
\text { la filosofía como } \\
\text { diálogo y como } \\
\text { enseñanza filosófica }\end{array}$ \\
\hline $\begin{array}{l}\text { Maite Larrauri (2001) de- } \\
\text { fiende la siguiente idea: en } \\
\text { la clase de filosofía se debe } \\
\text { comunicar, en parte, un co- } \\
\text { nocimiento filosófico y, en } \\
\text { parte, un deseo por el saber. } \\
\text { En este proceso se vincu- } \\
\text { lan pensamiento filosófico } \\
\text { y pensamiento común, aun- } \\
\text { que el primero cultiva nues- } \\
\text { tro inconformismo frente } \\
\text { al segundo. La propuesta } \\
\text { de Larrauri se denomina } \\
\text { maternaje y recoge, según } \\
\text { Gómez Mendoza (2003), } \\
\text { la perspectiva de Roland } \\
\text { Barthes. Desde este enfo- } \\
\text { que, la educación filosófi- } \\
\text { ca comprende: } 1 \text {. Prácticas } \\
\text { de educación o enseñanza } \\
\text { que transmiten los textos } \\
\text { filosóficos. } 2 \text {. Prácticas de } \\
\text { aprendizaje que comunican } \\
\text { la competencia filosófica. } 3 \text {. } \\
\text { Maternaje que comunica el } \\
\text { deseo de pensar por sí mis- } \\
\text { mos a los estudiantes. Las } \\
\text { fuentes de este trabajo son } \\
\text { los textos filosóficos occi- } \\
\text { dentales. Se acompaña de } \\
\text { una concepción del maes- } \\
\text { tro como aprendiz de filó- } \\
\text { sofo que sirve de mediador } \\
\text { entre los textos filosóficos y } \\
\text { los estudiantes. }\end{array}$ & $\begin{array}{l}\text { Esta perspectiva se asocia } \\
\text { con la tradición alemana. } \\
\text { En este contexto la filoso- } \\
\text { fía como disciplina escolar } \\
\text { se reinsertó en el Gimnasio } \\
\text { a partir de 1972. Según } \\
\text { Kledzik (1992) este inte- } \\
\text { rés por situar nuevamen- } \\
\text { te la filosofía en el proceso } \\
\text { formativo de los jóvenes } \\
\text { respondía a la demanda de } \\
\text { una formación que ayudara } \\
\text { a comprender los métodos } \\
\text { de producción de saber y } \\
\text { que, se espera, sirvan para } \\
\text { la vida. Lo que se enseñaría } \\
\text { en filosofía estaría orienta- } \\
\text { do al aprendizaje del filoso- } \\
\text { far vinculado fuertemente a } \\
\text { la tradición, a lo que se aso- } \\
\text { ciaría una formación en los } \\
\text { métodos de argumentación } \\
\text { filosófica. }\end{array}$ \\
\hline
\end{tabular}

Fuente: Paredes, 2017.
Tabla 2. Perspectivas del paradigma praxeológico para la enseñanza de la filosofía

\begin{tabular}{l} 
Paradigma praxeológico \\
En el paradigma praxeológico cabe propiamente la pro- \\
puesta del mismo Michel Tozzi de una filosofía asumi- \\
da como práctica y cuya meta es la formación filosófica \\
del público. \\
\hline Michel Tozzi: café filosófico \\
\hline La apuesta de este investigador es por una enseñanza \\
de la filosofía de corte práctico. Se defiende entonces \\
una inclinación a una educación para el filosofar, asu- \\
mida esta como ejercicio de la razón que se apoya en el \\
lenguaje natural para formular preguntas que generen \\
problemas a cualquier hombre y avanzar en su posible \\
resolución. Cabe anotar que Tozzi reconoce la dificultad \\
de lograr acuerdos entre enseñantes de filosofía acerca \\
de lo que entienden por filosofar, es por ello que lo máxi- \\
mo que se logra son acuerdos didácticos que permitirán \\
a los profesores construir un modelo didáctico del filo- \\
sofar (Tozzi, 2007).
\end{tabular}

Fuente: Paredes, 2017.

Considero que hay dos propuestas adicionales que pueden incluirse en el paradigma praxeológico y son: la educación filosófica como educación democrática y el Taller Filosófico. En la primera, tanto el maestro como estudiantes estarían en movimiento: la del maestro sería una acción sobre las acciones de los estudiantes en la que juega de manera plena como artista y filósofo (Álvarez, 2008; Bárcena, 1997). Como artista, debería desarrollar un conocimiento empático de los alumnos a través de sus interacciones con ellos, en este aspecto Dewey deja de lado cualquier pretensión de desarrollar un conocimiento objetivo de la infancia que orientaría de manera clara y precisa la práctica educativa. Como filósofo, debía mantener una actitud reflexiva permanente sobre el conocimiento a enseñar y sobre las disciplinas que le ayudarían a orientar su práctica -que serían la filosofía, la psicología y la sociología-, así como sobre los efectos de su práctica experiencial, en la que se buscaba "conectar su propio saber y experiencia y el conocimiento a enseñar con el flujo de la experiencia de los estudiantes" (Álvarez, 2008, p. 171). Desde este enfoque, el educador siempre estaría investigando acerca de su labor, aunque el énfasis no serán las materias de estudio ni los métodos adecuados para enseñarlas. En el centro de sus reflexiones se encuentra el análisis profundo de las fuerzas activas existentes en el joven, en calidad de posibilidades y promesas, que deben articularse a todas las herramientas que intervienen en el proceso educativo. El educador, en calidad de investigador de la educación, tendrá en su trabajo un nuevo compromiso que "está íntimamente 
ligado con el juicio y desarrollo de las condiciones, los materiales, las herramientas con las cuales se provocará, una vez más por la interacción con los poderes y preferencias existentes, la transformación deseada" (Dewey, 1989, p. 199). En cuanto al Taller de Filosofía, encontramos el trabajo de Oscar Breniffier (2005) en cuyo centro se encuentra la recuperación del papel formativo del debate y la figura del maestro como animador de la clase. Con esta apuesta, la enseñanza es vista como un laboratorio constante en la que el maestro siempre estará al acecho verificando la eficacia de los métodos que emplea para la enseñanza, máxime si consideramos que su función es lograr que los estudiantes trabajen y que eso se explicite en el debate constante entre ellos (Brenifier, 2005).

\section{¿Qué perspectiva de aprendizaje puede sustentar mi planeación?}

La propuesta de Klafki parte del supuesto que el aprendizaje es autónomo, pero para lograr constituirse como tal requiere de unas condiciones en el proceso de enseñanza: a) que se diseñe la propuesta didáctica partiendo del desarrollo psicomotor, cognitivo, estético, social y moral, de los intereses y del modo en que manejan los estudiantes las situaciones en general. b) La enseñanza debe abandonar los enfoques monolíticos en el tratamiento de los contenidos, de lo que se trata es de apropiar contenidos general que no cuentan, per se, con una secuencia que los ordena. Si bien, en términos cognitivos, encontramos propuestas sobre el aprendizaje como las de Jean Piaget (1976) en la que el desarrollo como un equilibrio progresivo explica el aprendizaje, Lev Vygotsky (1979) y su apuesta por una teoría histórico-cultural de la psiquis humana que también fue denominada sociointeraccionismo, o David Ausubel (1976) y su propuesta del aprendizaje significativo como aquel que busca abordar los contenidos desde las estructuras cognitivas de los estudiantes; no resultan tan potentes frente a lo que ofrece Jerome Bruner al pedagogo alemán.

Jerome Bruner, psicólogo estadounidense impulsó la ciencia cognitiva del siglo xx. En su estancia en Harvard, entre 1952 y 1972, desarrolló una teoría del aprendizaje en la que se ocupó de describir el proceso de aprender, los modos de representación y una revisión a las propuestas instruccionales. Con el tiempo pasó de ocuparse de la conducta a poner en el centro de la discusión el concepto de mente humana vista como una facultad que procesa, filtra y emplea la información que recibe del ambiente. El sujeto, a través de categorías, se apropia del mundo y fruto de sus intereses o necesidades construye representaciones de las cosas con base en las que actúa. En este momento cognitivo, Bruner (1997) asume que el aprendiz es capaz de construir conocimiento porque cuenta con unas categorías que le permiten apropiarse de la información que ofrece el ambiente que se va modificando. Lo anterior permite concebir el aprendizaje como un proceso en el aprendiz, quien asocia, construye y representa la realidad a través de lo enactivo, lo icónico y lo simbólico, teniendo preeminencia cada uno de estos modos de representación en las diferentes etapas de desarrollo humano (aquí sigue a Piaget).

A partir de la década de 1970, Bruner se interesó por las relaciones hombre y cultura, su obra La educación, puerta de la cultura (1997) da cuenta de este giro cultural en el que la educación es vista como un acontecimiento público en el que se intercambian, comparten y negocian significados. Así las cosas, en el análisis didáctica se recoge este giro y se parte del supuesto que la mente se construye culturalmente gracias a la educación; por ello, esta se enfoca en el trabajo sobre los actos de significado y la manera como se crean y comparten en el seno de una comunidad, aquí se pone en evidencia su proximidad a Vigostky. La preocupación ahora es por cómo se guía el proceso de enseñanza dando más responsabilidad al niño en el dominio de la tarea gracias a la interacción con sus pares; en el proceso formativo, siguiendo a Bruner, se debe apelar a las narraciones como recurso para comprender la manera de construir y compartir significados sobre el mundo y, finalmente, impulsar todas las estrategias que apunten a un aprendizaje recíproco.

\section{¿Qué perspectiva metódica puedo asumir en el diseño de las situaciones de enseñanza y aprendizaje?}

Si bien estos elementos se revisarán en el análisis y probablemente cambiarán, es importante identificar algunas propuestas que han pensado la asequibilidad del contenido filosófico y que están en correspondencia con las elecciones previas, por lo tanto se pueden incorporar en el diseño de la propuesta formativa en filosofía. 
Tabla 3. Metódicas para la enseñanza de la filosofía

\begin{tabular}{|l|l|}
\hline \multicolumn{2}{|l|}{ Propuestas de cine y filosofía } \\
\hline Grupo Embolic & Su apuesta es por una Filmosofía (Ferrer et al., 2011) se traduce en el diseño de \\
& fichas temáticas en donde se relacionan el filme, el filósofo y una novela a par- \\
& tir de “cuestiones" o preguntas, por ejemplo para hablar de Agustín se emplea \\
& el filme El Nombre de la Rosa y la novela homónima de Umberto Eco, de mo- \\
& do que ambas sirvan de puerta de ingreso al trabajo con la teoría agustiniana. \\
& El desarrollo de este trabajo lo podemos encontrar en su libro Primum videre, \\
& deinde philosophari: una historia de la filosofía a través del cine.
\end{tabular}

Propuestas sobre prácticas filosóficas

Este es un enfoque de la filosofía práctica o de la filosofía como forma de vida que se nutre de autores como Pierre Hadot (2009), actualmente hay seis grandes líneas de trabajo o familias metodológicas en el campo de las prácticas filosóficas: filosofía para niños, cafés filosóficos, talleres filosóficos, diálogos neosocráticos, orientación filosófica y filosofía en las organizaciones. De todas ellas reiteramos la pertinencia de los cafés filosóficos, talleres filosóficos, los diálogos neosocráticos y cierto tipo de orientaciones filosóficas. Del café filosófico y del taller filosófico ya hice referencia antes, por lo cual ampliaré las dos restantes.

\begin{tabular}{l|l} 
Diálogos neosocráticos & Se introdujeron en el contexto alemán con el fin de mejorar la enseñanza de la
\end{tabular} filosofía y recuperar la línea kantiana de aprender a filosofar. Los impulsadores de esta propuesta fueron Leonard Nelson y Gustav Heckmann y su enfoque era formar en el filosofar siguiendo el modo de diálogo de Sócrates. Cuenta, para su desarrollo, con unas reglas muy precisas y se emplea actualmente en organizaciones, empresas, etc.

Orientación filosófica

Se hace un uso terapéutico de la filosofía que pone al filósofo en un lugar equivalente al de un psicólogo clínico o un médico y aleja a la filosofía de la dimensión teórica de la universidad o la escuela. Uno de los autores de mayor trascendencia es Lou Marinoff, quien ha sido el primero en emplear el concepto práctica filosófica en una perspectiva amplia e incluye metodologías diversas en las que se trabaja con individuos, grupos y organizaciones.

\section{Propuestas de escritura filosófica}

Dos autores que han pensado ampliamente el vínculo entre escritura y formación filosófica en el marco de la didáctica de la filosofía han sido Michel Tozzi, en Francia, y Miguel Gómez Mendoza, en Colombia.

Michel Tozzi (2003)

El aprendizaje del filosofar implica para Tozzi el manejo de la información, la problematización, la conceptualización y la argumentación. Las dimensiones del aprendizaje del filosofar se centran en el progreso del aprendizaje del alumno y se desarrollan en relación con la lectura, la expresión verbal y la escritura. De lo que se trata el trabajo filosófico es de un vínculo entre los conceptos, el modo en que estos surgen y tienen sentido, y que las elaboraciones en torno a ello requieren de pruebas que se ofrezcan discursivamente.
Miguel Gómez Mendoza $(2003,2005)$

Se ha interesado por disertación y el comentario de texto filosófico. La disertación se constituye en una herramienta poderosa para desarrollar la capacidad reflexiva de los alumnos. Se trata de un texto en el que se articulan argumentos sobre un tema o pregunta y que permite evaluar el índice de comprensión y creación de los estudiantes, fruto de sus búsquedas en el corpus teórico, usualmente comprende las siguientes partes: 1. Leer un tema. 2. Plantear un problema. 4. Analizar los elementos del problema. 5. Construir un plan de escritura. 6. Demostrar las ideas, lo que implica refutar las propuestas contrarias.

En el caso del comentario de texto filosófico, la meta es extraer el interés filosófico de un texto y continuar con su estudio ordenado. Debe contar con introducción, desarrollo y conclusión.

Fuente Paredes, 2017. 


\section{El análisis didáctico como eje del curso el curso Didáctica II (Filosofía) en la Universidad de Antioquia: síntesis del proceso}

El curso que describiré se denominó Didáctica II (Filosofía), materia obligatoria en el programa Licenciatura en Filosofía, y se desarrolló en la Seccional Oriente de la Universidad de Antioquia durante todo el semestre 2013-II, y contó con varios momentos:

1. Revisión de aspectos generales sobre la enseñanza de la filosofía en Colombia: de la mano de Óscar Saldarriaga (2008) se evaluaron las transformaciones históricas en la enseñanza de la filosofía en Colombia durante el siglo XIX, así como los retos para el siglo Xx. Luego de lo cual se avanzó en un trabajo con la Ley Uribe de 1903, la Ley 115 de 1994, la resolución 2343 de 1996 y las Orientaciónes de 2010. La finalidad de este proceso era evaluar el marco general de sentido para la formación filosófica en el nivel medio de educación en Colombia así como los alcances y tensiones en torno a la formación filosófica en el país.

2. Aproximación a diferentes perspectivas filosóficas: se exploraron las visiones de autores europeos como Immanuel Kant, Wolfgang F. Hegel, John Dewey, Michel Onfray, Michel Tozzi; autores latinoamericanos como Walter Kohan, y autores colombianos como Diego Pineda, Germán Vargas Guillén y Adolfo León Gómez. La finalidad de este proceso era invitar al estudiante a situarse en ciertas discusiones sobre el estatuto disciplinar de la filosofía, su función en la vida humana y las implicaciones de asumir una postura filosófica al momento de planear una propuesta formativa. Con este recorrido se impulsaba a los estudiantes a que revisaran su formación disciplinar y las fortalezas de esta.

3. Análisis de propuestas didácticas para la enseñanza de la filosofía: se trabajaron diferentes perspectivas didácticas y metódicas sobre la enseñanza de la filosofía, por ejemplo: se analizaron los trabajos de Miguel Ángel Gómez, Luz Gloria Cárdenas, Michel Tozzi, Mathew Lipmann, del grupo Embolic, etc. Autores que ofrecían un panorama bastante amplio sobre las discusiones en torno a la enseñanza de la filosofía y que, de manera simultánea, introducían al estudiante en la familiarización con ciertas propuestas metódicas como el Foro de Filosofía, el uso del cine para la enseñanza de la filosofía, la Comunidad de Indagación, la disertación filosófica, los ambientes virtuales de aprendizaje, los diccionarios filosóficos, etc.

4. Profundización en la perspectiva de Wolfgang Klafki: esto se realizó de manera transversal durante el semestre. Inicialmente se ofrecía a los estudiantes una reconstrucción de la propuesta didáctica del autor y se enunciaban las preguntas del análisis didáctico que eran retomadas a lo largo del desarrollo de cada componente, por ejemplo las preguntas 1, 2 y 3 del análisis se relacionaban con los debates sobre la filosofía y el sentido de esta en la educación colombiana; la pregunta 4 se nutría también de estos debates, pero se agregaba la reflexión sobre la enseñanza de la filosofía; finalmente, la pregunta 5 se vinculaba con el tema de las metódicas. También este trabajo contó con una aproximación, muy general, a la teoría del aprendizaje significativo desde Juan Ignacio Pozo y Ángel Díaz Barriga, así como la recuperación de los saberes con que contaban los estudiantes sobre este campo.

5. Trabajo de campo y productos: como una de las demandas de la propuesta de Klafki es el trabajo con ejemplos, se dispuso una serie de orientaciones para impulsar la experiencia en campo de los futuros licenciados en filosofía. Lo primero fue construir una guía en la que hice unas adaptaciones a las preguntas de Klafki y diseñé un trabajo de campo en instituciones educativas cuyo fin era recuperar la experiencia de docentes de filosofía en ejercicio en el Oriente Antioqueño. Lo segundo, acompañar la construcción por parte de los estudiantes de una narrativa con apoyos audiovisuales sobre los hallazgos en contraste con las orientaciones del pedagogo alemán. La meta de esta actividad era mostrar que, si bien los profesores entrevistados no necesariamente conocían la propuesta didáctica de Wolfgang Klafki, si evidenciaban ciertos elementos afines a él o también se distanciaban. Las preguntas que guiaban este trabajo son las que se detallan en la tabla 4. 
Tabla 4: Preguntas guía de trabajo de campo

\begin{tabular}{|c|c|}
\hline Exploración general & $\begin{array}{l}\text { Con el docente de filosofía realizarán el siguiente trabajo: } \\
\text { - Indagación sobre el perfil profesional. } \\
\text { - Pregunta acerca de la concepción de filosofía con que contaba al momento de egresar } \\
\text { de su carrera. Se suma a esto la concepción de la enseñanza de la filosofía que tam- } \\
\text { bién tenía en tal momento. } \\
\text { - Pregunta acerca de la concepción de filosofía con que cuenta en este momento en su } \\
\text { ejercicio profesional. Concepción de la finalidad que tiene la filosofía actualmente. }\end{array}$ \\
\hline $\begin{array}{l}\text { Desempeño en el } \\
\text { escenario educativo }\end{array}$ & $\begin{array}{l}\text { Con el docente de filosofía realizará el siguiente trabajo: } \\
\text { - ¿Desde qué enfoque conceptual realiza su planeación? } \\
\text { - ¿Con qué formatos diseña su trabajo anual y por periodos? } \\
\text { - ¿Qué contenidos desarrolla durante el año escolar? ¿Qué competencias pretende } \\
\text { desarrollar? } \\
\text { - ¿Qué estrategias de aprendizaje y enseñanza emplea para la ejecución de su propues- } \\
\text { ta académica? } \\
\text { - ¿Cómo evalúa el proceso de los estudiantes? } \\
\text { - ¿Qué dificultades encuentra en su trabajo académico? }\end{array}$ \\
\hline $\begin{array}{l}\text { Fuera del escenario } \\
\text { educativo }\end{array}$ & $\begin{array}{l}\text { De acuerdo al formato que trabaja el docente con quien ha entablado relación e incorpo- } \\
\text { rando el enfoque de Klafki realizará su planeación para entregar en el curso. }\end{array}$ \\
\hline
\end{tabular}

Fuente: Paredes, 2017.

Con todas estas herramientas los estudiantes debían enfocarse en el diseño de una propuesta formativa en enseñanza de la filosofía a partir del análisis didáctico propuesto por Wolfgang Klafki con la siguiente instrucción: 1. El enfoque de aprendizaje a recuperar era el aprendizaje significativo. 2. En la pregunta por la asequibilidad del contenido se debían vincular tanto los elementaria que expone el autor como dos recursos metódicos: el cine y un sitio web que debían diseñar. Presento entonces, en la tabla 5, una síntesis de una de las propuestas formativas o unidad didáctica como producto final de este curso.

Tabla 5. Síntesis ejemplo de planeación de propuesta formativa en filosofía del estudiante Óscar Osorio

\begin{tabular}{|l|l|}
\hline \multicolumn{2}{|c|}{ Ficha resumen del análisis didáctico llevado a cabo por el estudiante Óscar Daladier Osorio } \\
\hline \multicolumn{1}{|c|}{ Tema } & \multicolumn{1}{|c|}{ La ética del cuidado de sí en Michel Foucault } \\
\hline $\begin{array}{l}\text { Perspectiva filosófica } \\
\text { y didáctica }\end{array}$ & $\begin{array}{l}\text { El estudiante se propone transformar en formativa La ética del cuidado de sí desde la pers- } \\
\text { pectiva de Michel Foucault. Para ello la sitúa en el orden moral y político vinculado a la } \\
\text { pregunta por los modos en que los estudiantes gobiernan su propia vida y los ámbitos en } \\
\text { que esta se desarrolla. Se acoge una perspectiva edificante de la filosofía como punto de } \\
\text { partida para la planeación, por ello se nutre la propuesta de Foucault con las prácticas de } \\
\text { libertad que experimentan los estudiantes con quienes se planea desarrollar el trabajo. }\end{array}$ \\
\hline $\begin{array}{l}\text { Grupo de aprendizaje y } \\
\text { contexto sociocultural }\end{array}$ & $\begin{array}{l}\text { La población para la que fue pensada la propuesta eran estudiantes de undécimo del mu- } \\
\text { nicipio de La Unión (Antioquia), cuya edad puede oscilar entre los 15 y los 18 años. Se } \\
\text { seleccionó esta población porque se esperaría que cuenten con cierta familiaridad con } \\
\text { el trabajo filosófico y algunas capacidades asociadas a su estudio. La meta formativa es } \\
\text { fortalecer las cualidades ganadas por ellos y, además, llevar a cabo un análisis crítico de } \\
\text { la performatividad, los discursos y las instituciones que giran en torno al cuerpo, las cos- } \\
\text { tumbres, las relaciones intersubjetivas, el poder etc., buscando estimular nuevas formas } \\
\text { de relacionarse con los otros, con las cosas y con ellos mismos. }\end{array}$ \\
\hline
\end{tabular}


Pregunta 1: Significado ejemplar del contenido
Para su desarrollo, el estudiante Osorio inició con la idea de práctica filosófica a partir del siglo XviII desde Michel Foucault. El texto que sirvió de base a este momento es Las tecnologías del yo, seleccionado porque su temática permite comprender qué es una actitud crítica y el por qué se asume como una nueva manera de filosofar, un filosofar que cambia la vida y que permite construirla de otros modos. Luego de ello, apropiando el concepto de estética de la existencia, propuso un análisis sobre discursos, rituales e instituciones, a partir de lo cual se tratarían de pensar prácticas de libertad y se plantearía aquí un problema actual a partir del cual se podría desarrollar, a su modo de ver, el tema de la ética del cuidado de sí. Interesaba que los estudiantes se apropiaran de la idea de que la performatividad, los discursos y las instituciones que determinan su vida y los ámbitos en los que esta se desarrolla no constituyen entidades originariamente dadas, por ello se puede reflexionar sobre ella y transformarlas.
Preguntas 2 y 3 : importancia para el presente y el futuro

Para responder a estas dos preguntas se valió el estudiante Osorio del mismo Foucault, especialmente de su propuesta genealógica y arqueológica de la historia. Toda vez que esta se enfocó en ofrecer los elementos para que podamos comprender nuestro presente y presentar unos modos de proceder que pueden ser emulados por los estudiantes en formación. La incorporación de estos modos puede ayudar al individuo a concebirse a sí mismo como un ser libre, es decir, como un ser que puede ejercer cierto dominio sobre sí, que puede configurarse a sí mismo de cierta manera, rompiendo con la idea de ser pasivo. Lo anterior permitiría apropiar los contenidos del concepto o categoría de este trabajo, a saber: ética del cuidado de sí. Lo que sitúa al contenido en la vida futura del educando ya que se espera que logre hacerlo florecer en otras situaciones que enfrente, decisiones que tome y discursos que reconozca.

Pregunta 4: estructura del contenido

Para definir la estructura se apeló al desarrollo de la obra de Foucault y a la legislación vigente en Colombia en el campo de la enseñanza de la filosofía. El estudiante observó la correspondencia entre la propuesta moral y política de Foucault con el ámbito político que está en las Orientaciones pedagógicas para la filosofía en la educación media (2010) en cuyo centra está la discusión por la libertad, el poder y la voluntad. A renglón seguido, el estudiante acudió al enfoque del aprendizaje significado para clasificar los momentos del proceso así: 1) momento preinstruccional: en él se revisarían los presupuestos y el sentido de la acción filosófica, se explorarían saberes previos sobre la libertad, el poder y la voluntad. 2) Momento coinstruccional: se llevaría a cabo una introducción general de la obra de Foucault (marco de emergencia de su propuesta) y, a partir de analogías, se rastrearían semejanzas entre las condiciones de su época que hicieran posible sus ideas y las condiciones presentes de los estudiantes en formación. Hecho lo anterior, se procedería al abordaje de la propuesta de investigación arqueológica y genealógica de investigación filosófica o crítica que deja planteada Foucault, así como sus ideas y conceptualizaciones en torno al cuidado de sí, a la cuales se ligan, a su vez, conceptos como los de libertad, modernidad, askesis, parrhesía, kinismo, entre otros, que son importantes para entender esa idea de la ética como cuidado de sí. 3) Momento posinstruccional: aquí se trataría de analizar el problema actual en torno a los discursos sobre el cuerpo y el género, a partir de las herramientas adquiridas con el estudio de las categorías e ideas en torno al cuidado de sí de Michel Foucault; esta actividad se llevaría a cabo -para decirlo en los términos de Klafki- de un modo ejemplar (elementaria), esto es, a partir de experiencias y situaciones cotidianas de los individuos que permitan dilucidar las cuestiones problemáticas que se pretenden abordar. Lo que interesa analizar de dicho problema es, como ya se ha dicho, la performatividad, los discursos y las instituciones que giran en torno a ellos y que determinan la forma en que nosotros los vemos y pensamos; esto con el fin de dilucidar otros modos de ser que nos permitan transformarnos a nosotros mismos y, con ello, a nuestra forma de relacionarnos con las cosas y con los otros. 
Pregunta 5: asequibilidad del contenido
Esta es la parte que corresponde a la recuperación de paradigmas para la enseñanza de la filosofía como el problemático y para metódicas referidas al uso del cine para la enseñanza de la filosofía (La grande bouffe, del director Marco Ferreri, ésta permite dilucidar formas diferentes a las convencionales de relacionarse con uno mismo, con los otros y con las cosas), las preguntas abiertas, la clase como laboratorio conceptual en la que se analizan discursos de dirigentes e informes de experimentos sociales relacionados con el tema, la escritura de sí. Por ello, las experiencias de los estudiantes y sus saberes previos sirven como material inicial para el análisis de los conceptos libertad, voluntad y poder y como puente para traer a escena tanto la estética de la existencia como la ética del cuidado de sí. Con lo cual se cumple una de las exigencias esenciales del análisis klafkiano: que lo categorial se transforma en horizonte de sentido del estudiante y se incorpora a través de la vivencia. Finalmente, el estudiante Óscar Osorio aclara que se valdrá de formas de lo fundamental y lo ejemplar (elementaria), para el manejo de la asequibilidad del contenido.

Fuente: Paredes, 2017. Curso Didáctica II (Filosofía).

\section{Conclusiones}

La didáctica crítico-constructiva es una perspectiva de síntesis entre las propuestas de formación formal y de formación material que supera la dicotomía entre contenidos y cultivo de las capacidades de los educandos. Con ello, Klafki le ofrece al didacta herramientas para que pueda definir criterios claros que orienten el diseño del encuentro formativo en diferentes escenarios educativos, sin instrumentalizar su quehacer y sin reducir los contenidos a secuencias acabadas de información.

La propuesta klafkiana exige al didacta despojarse de cualquier pretensión de neutralidad en la planeación. Al recuperar la noción de Bildung y acompañarla de los cambios propuestos por la tradición crítica, este enfoque didáctico hace visible el vínculo entre los contenidos, el horizonte de mundo que se ofrece al estudiante y el cultivo de sus capacidades solidarias y de autogestión, cuya meta sea la superación de la desigualdad y de las prácticas alienantes.

El análisis didáctico sitúa al didacta como productor de saber, lo anterior porque cada planeación ha exigido un tratamiento de la disciplina a enseñar, de las características de la población a atender, de las condiciones sociales que modelan la situación educativa, de la articulación del contenido con los fines individuales y sociales, de las condiciones específicas del aprendizaje y de la pregunta por los canales que harían asequible el contenido.

Los licenciados en filosofía en formación o ejercicio pueden encontrar en Klafki una alternativa para nutrir su trabajo, especialmente en los niveles de educación básica y media en Colombia. El análisis didáctico es una herramienta que ayudará en la selección de los contenidos a enseñar y de las capacidades a cultivar en el educando, pues exigirá que siempre se dé cuenta del potencial formativo de ese contenido y del modo en que este se vincula, a través de ejemplos, a la cotidianidad de los estudiantes.

\section{Referencias}

Álvarez, A. (2008). La filosofía como pedagogía. En: G. Hoyos V. (ed.). Filosofía de la Educación (pp. 157-177). Madrid: Trotta.

Arnaiz, G. (2004). Oscar Brenifier: el mago de los talleres filosóficos. Revista ETOR, (3), 9-16.

Arnaiz, G. (2007). Relevancia de las aportaciones de Pierre Hadot y Michel Onfray para la Filosofía Práctica. A parte Rei. Revista Filosófica, (52), 1-9.

Ausubel, D. (1976). Psicología educativa: un punto de vista cognoscitivo. México: Editorial Trillas.

Bárcena, F. (1997). El oficio de la ciudadanía. Introducción a la educación política. España: Paidós.

Brenifier, O. (2005). Enseñar mediante el debate. México: Edere.

Bruner, J. (1997). La educación, puerta de la cultura. Madrid: Visor.

Dewey, J. (1989). The Need for a Philosophy of Education. En: J. Dewey. Later works: IX (pp. 194-204). Carbondale: Southern Illinois University Press.

Ferrer, A.; García Raffi, X.; Hernández, I.; Dobon, F. y Lerma, B. (2006). Primum videre, deinde philosophari: una historia de la Filosofía a través del cine. Valencia, España: Institucio Alfons el Magnanim.

Ferrer, A.; García R., X.; Hernández, I.; Dobon, F. y Lerma, B. (2012). Notas para una Fil(m)osofía. En: L. Cárdenas y C. Restrepo (eds.). Didácticas de la filosofía. Experiencias, instrumentos y métodos (vol. 2. pp. 187214). Medellín, Colombia: Universidad de Antioquia, Ediciones San Pablo. 
Foucault, M. (2008). La hermenéutica del sujeto. Argentina: Fondo de Cultura Económica.

Gaitán, C.; López, E.; Quintero, M. y Salazar, W. (2010). Orientaciones pedagógicas para la filosofía en la educación media. Bogotá: Ministerio de Educación Nacional.

Gómez M. (2003). Introducción a la didáctica de la filosofía. Pereira, Colombia: Universidad Tecnológica de Pereira.

Gómez M. (2005). Didáctica de la disertación en la enseñanza de la filosofía: métodos y procedimientos. Bogotá: Magisterio.

Hadot, P. (2009). La filosofía como forma de vida. Barcelona: Alpha Decay.

Klafki, W. (ed.). (1970). Funk-Kolleg Erziehungswissenschaft. Eine Einführung in drei Bänden. Frankfurt am Main: Fischer.

Klafki,W.(1972). Das pädagogische Problem des Elementaren und die Theorie der kategorialen Bildung. Weinheim: Beltz.

Klafki, W. (1995). Didactic analysis as the core of preparation of instruction. Journal of Curriculum Studies, 27(1), 13-30.

Klafki, W. (1975). Studien zur Bildungstheorie und Didaktik. Weinheim: Beltz.

Klafki, W. (1985a). Neue Studien zur Bildungstheorie und Didaktik. Weinheim: Beltz.

Klafki, W. (1985b). La importancia de las teorías clásicas de la educación para una concepción de la educación general hoy. Educación (Tubingen), 36(291), 105-127.

Klafki, W. (1991). Sobre la relación entre didáctica y metódica. Revista Educación y Pedagogía, 2(5), 85-108.
Kledzik, S. (1992). Les recherches contemporaines en didactiquede la philosophie. Recuperado de: http://www. philotozzi.com

Larrauri, M. (2001). La educación filosófica. En: C. Lomas (comp.). ¿Educar o segregar? Materiales para la transformación de la educación secundaria (pp. 144-154). Bogotá: Coomagisterio, Universidad Nacional.

Marinoff, L. (2001). Philosophical Practice. Nueva York: Academic Press.

Onfray, M. (2008). La sabiduría de la antigüedad. Contrahistoria de la filosofía, I. Barcelona: Anagrama.

Piaget, J. (1976). La toma de conciencia. Madrid: Morata.

Putnam, H. (1997). La herencia del pragmatismo. Barcelona: Paidós.

Rorty. R. (2010). La filosofía y el espejo de la naturaleza. Madrid: Cátedra.

Runge, A. (2008). Ensayos sobre pedagogía alemana. Bogotá: Universidad Pedagógica Nacional, Museo Pedagógico Colombiano.

Sharp, A. y Splitter, L. (1996). La otra educación. Filosofía para niños y la comunidad de indagación. Buenos Aires: Manantial.

Tozzi, M. (2003). Aprendre a philosoher. Le role de la discussion ET dês formes diversifees d'ecriture. Recuperado de: http://www.pratiquesphilosophique.com

Tozzi, M. (2007). Sobre la didáctica del aprendizaje del filosofar. Diálogo Filosófico, 68, 207-215.

Vygotsky, L. (1979). El desarrollo de los procesos psicológicos superiores. Barcelona: Crítica.

Weniger, E. (1962). Didaktik als Bildungslehre. Weinheim: Beltz. 